\title{
Supersonic cloud collision. I.
}

\author{
S. Anathpindika ${ }^{1,2}$ \\ 1 School Of Astronomy \& Physics, Cardiff University, 5-The Parade, UK \\ 2 Indian Institute Of Astrophysics, 2nd Block - Koramangala, Bangalore 560 034, India \\ e-mail: Sumedh.Anathpindika@astro.cf.ac.uk, sumedh-a@iiap.res.in
}

Received 29 January 2009 / Accepted 27 May 2009

\section{ABSTRACT}

\begin{abstract}
Context. It has long been suggested that shocks might play an important role in altering the form of the interstellar medium (ISM). Shocks enhance gas density and sufficiently dense regions may become self gravitating. Potential star-forming clouds within larger molecular clouds, move randomly at supersonic speeds and a collision between them, is highly inelastic.

Aims. Depending on the precollision velocity, colliding molecular clouds produce a slab that is either shock-compressed or pressureconfined. In a sequel of two Papers (I and II), we simulate molecular cloud collision and investigate the dynamical evolution of such slabs. Shocked slabs appear susceptible to hydrodynamic instabilities and, in the present Paper (I), we study the effect of strong shear between slab layers on the dynamic evolution of a shock compressed gas slab. Head-on and off-centre cloud collisions have been examined in this work. Self gravity is included in all our simulations.

Methods. Simulations presented were performed using the smoothed particle hydrodynamics (SPH) numerical scheme. Individual, precollision clouds are modelled as pressure-confined Bonnor-Ebert spheres. However, for simplicity the thermodynamic details of the problem are simplified and the gas temperature is evolved simply by a barytropic equation of state. Obviously, the gas suffers to some extent from thermal inertial effects. However, we note that the dynamical timescale is much smaller than the local sound crossing time so that any such effects should have minimum influence.

Results. Strong shocks are highly radiative. Thus a highly supersonic cloud collision produces a cold, roughly isothermal shock compressed gas slab. We find that the shocked slab is susceptible to dynamical instabilities like the gravitational instability, KelvinHelmholtz (KH) instability and the non linear thin shell instability (NTSI). Rapid growth of instabilities within the slab produces structure in it. The NTSI competes with the gravitational instability and the fate of the shocked slab apparently depends on the relative dominance of either of the two instabilities. Dominance of the NTSI causes turbulent mixing between slab layers and dissipates internal energy. Eventually the slab collapses to form a thin elongated body, aligned with the collision axis, and star formation may commence in it. Our hydrodynamical models discussed here suggest that, high-velocity cloud collisions may be a viable mechanism for the formation of observed filamentary structure in the ISM.
\end{abstract}

Key words. hydrodynamics - shock waves - ISM: clouds - instabilities - star: formation

\section{Introduction}

Spiral shock waves sweep up matter in galactic arms and produce large-scale structure and dust lanes (Roberts 1969; Fleck 1992; Heitsch et al. 2006; Elmegreen 2007). Often MCs are themselves filamentary, and such clouds, may form out of gravitationally unstable gas sheets (Nagai 1998). Detailed studies of molecular line profiles of MCs have revealed that the matter within them is highly unevenly distributed and gives them a clumpy appearance, which has led some to suggest a fractal model of MCs (Elmegreen 1997).

Schneider \& Elmegreen (1977) performed one of the first studies of large-scale filamentary structures in the local neighbourhood. Now with improved technology, it is possible to map MCs in greater detail. For instance, dense filamentary structures have been observed in the Perseus region where there is evidence of ongoing star formation (Hatchell et al. 2005). The Orion integral Filament (OIF) in the Orion A region has been studied in great detail and several young stellar objects (YSOs) have been reported to be embedded in it (Chini et al. 1997; Johnstone \& Bally 1999).

Structure within MCs appears to be hierarchical, and the densest regions are gravitationally bound; see for instance, Larson (1981) and Elmegreen (2007). Elongated molecular and atomic structures are common occurrences in the ISM and several possible formation mechanisms have been suggested (cf. Kulkarni \& Heiles 1987). One is the dynamical interaction between turbulent fluid flows within MCs (Klessen \& Burkert 2000; McLow \& Klessen 2004). Turbulence decays rather quickly on a timescale equal to the dynamical period of evolution of the cloud. The importance of turbulence in star formation has been highlighted by several workers (e.g. Sasao 1973; Klessen et al. 2000; Klessen \& Burkert 2001, and references therein). Gas slabs resulting from the collision of stellar winds from binary systems expanding at supersonic speeds become thermally unstable due to rapid cooling, also turbulent motion within shells makes them unstable to shearing instabilities (Stevens et al. 1992). Similarly, powerful ionising radiation emitted by young OB associations or supernova remnants sweeps up gas and forms a dense shell that is gravitationally and thermally unstable (Strickland \& Blondin 1995; Dale et al. 2007).

Analogous to the random motion of smaller clouds, larger MCs (giant molecular clouds, GMCs) in galactic arms also exhibit random motion. The line width of $\mathrm{CO}$ emission from GMCs suggests that clouds have a random velocity dispersion typically of a few $\mathrm{km} \mathrm{s}^{-1}$, superposed on their systematic galactic motion (Spitzer 1968; Solomon et al. 1987). GMCs are largely 
supported by internal turbulence and have lifetimes of about a few tens of Myrs, before being dispersed by stellar feedback (e.g. McLow 1999; Padoan \& Nordlund 1999; Klessen et al. 2000; Blitz et al. 2007).

Smaller clouds within GMCs themselves have turbulent velocities of $\sim 1-2 \mathrm{~km} \mathrm{~s}^{-1}$ on a parsec scale (e.g. Larson 1981; Elmegreen 2000). Collision between such clouds, moving randomly with supersonic speeds is an efficient mode of dissipating kinetic energy. High-velocity cloud collision is a violent phenomenon that might trigger star formation in the resulting composite object. The gas bodies resulting from dynamically interacting fluid masses already contain the necessary seeds of dynamic instability. Magnetic field threading the GMCs is generally weak, a few $\mu \mathrm{G}$ only, and does not seem to play any significant role in damping turbulence within GMCs. However, the magnetic field strength could increase dramatically due to postshock enhancement of gas density, which in turn may cause further compression of the shocked gas (e.g. Hartmann et al. 2001). Low-velocity MC collision produces gravitationally unstable gas slabs (e.g. Bhattal et al. 1998).

The problem of colliding clouds has been studied numerically in the past, for instance by Stone (1970a,b), Hausman (1981), Lattanzio et al. (1985) and Hunter et al. (1986), and more recently by Bhattal et al. (1998) and Klein \& Woods (1998), among others. Stone (1970a) studied this problem in one dimension by simulating MCs as planes having infinite spatial extent and found that the clouds coalesced post collision and, the resulting gas slab then expanded. The same experiment in two dimensions also yielded a similar result (Stone 1970b). Hausman (1981) performed particle simulations of head-on and off-centre cloud collisions. However, the results were corrupted by particle penetration during collision.

Later Hunter et al., in their grid simulation of colliding flows, found that such collisions produced slabs that, according to them, were unstable to Rayleigh-Taylor like instability, but could not establish the exact nature of this instability. A similar conclusion was drawn by Stevens et al. (1995) and Klein \& Woods (1998). However, both found evidence of non linear growth of instabilities starting in thin shells within the slab. In fact, the latter suggested that the non linear thin shell instability (NTSI) occurs in the shocked slab, resulting from cloud collisions.

In this paper we demonstrate the occurrence of this instability in our smoothed particle hydrodynamics (SPH) simulations of highly supersonic $\left(10 \mathrm{~km} \mathrm{~s}^{-1}\right.$ to $\left.20 \mathrm{~km} \mathrm{~s}^{-1}\right)$ head-on cloud collision. For the purpose of this work, we used clouds with only token masses, a few orders of magnitude smaller than that of real star-forming clouds. Although we test one model with slightly massive clouds, even their masses are still modest. Limited computational resources create a major hurdle when using a large number of particles, since increasing the precollision cloud mass without increasing the number of gas particles will simply raise the minimum resolvable mass and, at the same time reduce spatial resolution. The precollision velocity of individual clouds, however, is representative of the dispersion velocity of clouds in the galactic plane. The possible effect of the SPH artificial viscosity $(\mathrm{AV})$ on the growth rate of the NTSI is left for a future paper.

The plan of the paper is as follows. In Sect. 2 we briefly describe the SPH code employed for our cloud collision experiments. In Sect. 3 we discuss the procedure for setting up the initial conditions for cloud collision experiments and briefly discuss the models tested. In Sect. 4 we discuss the NTSI and simulation results and conclude in Sect. 5. Since the column density spans roughly eight orders of magnitude, all column den- sity plots presented here are plotted on a logarithmic scale. The column density is measured in $M_{\odot} \mathrm{pc}^{-2}$ and the distance in pc.

\section{Smoothed particle hydrodynamics (SPH)}

SPH is a Lagrangian interpolation scheme used to treat complicated problems in astrohydrodynamics. Under the scheme, the fluid being investigated is treated as an ensemble of a large number of particles or fluid markers. The particles in this case are not point masses in the strictest sense, but have a finite spatial extent (Gingold \& Monaghan 1977; Lucy 1977).

All the simulations presented here were performed using the DRAGON SPH code. It is a well-tested code, the relevant details of which can be found in (Goodwin, Whitworth \& WardThompson 2004). It uses the Barnes-Hut tree for gravity calculation $\left(\theta_{\text {crit }} \sim 0.45\right)$ and also includes quadrupole moments of remote leaf cells. The forces are integrated using a secondorder Runge-Kutta integration scheme. Each particle has $(50 \pm 5)$ neighbours. The code employs a multiple particle time-stepping scheme rather than global time-stepping and uses the standard SPH artificial viscosity.

Extremely dense agglomerations of particles in the computational domain are replaced by sinks, characterised by a fixed density, $\rho_{\text {sink }}$, and radius, $R_{\text {sink }}$. The concept of a sink particle was first introduced in SPH by Bate, Bonnell \& Price (1995). The rest of the gas particles in the computational domain interact with the sink only through gravity. SPH particles coming within a predefined radius, called the sink radius $\left(R_{\text {sink }}\right)$, are accreted by it, provided particles being accreted satisfy certain criteria (Bate, Bonnell \& Price 1995). In all the simulations presented here, the sink density is held fixed at $\rho_{\text {sink }}=10^{-12} \mathrm{~g} \mathrm{~cm}^{-3}$, and $R_{\text {sink }}$ is chosen so that the initial sink mass, $M_{\text {sink }}$, is comparable to the minimum resolvable mass in the simulation, $M_{\min }$. Now,

$M_{\text {min }}=N_{\text {neibs }} \cdot m_{i}$

where $N_{\text {neibs }}$ is the number of nearest neighbours of an SPH particle, $m_{i}=\frac{M_{\text {cld }}}{N_{\text {gas }}}, N_{\text {gas }}$ the number of gas particles in the simulation, and $M_{\text {sink }}=\frac{4}{3} \pi R_{\text {sink }}^{3} \rho_{\text {sink }}$. In view of the above-mentioned requirement,

$R_{\text {sink }} \sim\left(\frac{N_{\text {neibs }} M_{\text {cld }}}{4 N_{\text {gas }} \rho_{\text {sink }}}\right)^{1 / 3}$.

In the present work, the sink particle represents a protostellar core.

\section{Numerical experiments}

\subsection{Setting the initial conditions (ICS)}

We first describe the scheme for assembling particles within a molecular cloud (MC). The MC is modelled as an external pressure-confined isothermal sphere, characterised by its dimensionless radius, $\xi$, and it has been well-established that only those spheres having radii smaller than a critical radius, $\xi_{\text {crit }}=$ 6.45 , can remain in equilibrium (Chandrasekhar 1939). For our purposes, we choose $\xi_{B} \equiv \xi=3$, which ensures that the sphere is supported against self gravity.

For simplicity, we further assume that gas within this sphere is primarily composed of molecular hydrogen and small quantities of helium (relative abundance of 0.1 relative to hydrogen). If 
$\bar{m}$ is the mean molecular mass of a gas particle, then the isothermal sound speed $a_{0}$ is defined as

$a_{0}=\left(\frac{k_{\mathrm{B}} T_{\mathrm{cld}}}{\bar{m}}\right)^{\frac{1}{2}}$.

Here $k_{\mathrm{B}}$ and $T_{\text {cld }}$ respectively, are the Boltzmann constant and uniform gas temperature in the sphere. The mass interior to this sphere, $M\left(r_{B}\right)$, is

$M_{\text {sphere }} \equiv M\left(r_{B}\right)=4 \pi R_{0}^{3} \rho_{c}\left(\xi^{2} \frac{\mathrm{d} \psi}{\mathrm{d} \xi}\right)_{\xi_{B}}$,

where $\psi(\xi)$ is called the Emden function (Chandrasekhar 1939) and the physical radius of the sphere, $r_{B}=R_{0} \xi_{B}, R_{0}$ having the dimension of length is a distance scale factor, and defined as

$R_{0}=\frac{a_{0}}{\left(4 \pi G \rho_{\mathrm{c}}\right)^{\frac{1}{2}}}$.

Taking the ratio of $M_{\text {sphere }}$ with $r_{B}$, we get

$$
\begin{gathered}
\frac{M_{\text {sphere }}}{r_{B}}=\frac{a_{0}^{2} \mu\left(\xi_{B}\right)}{G \xi_{B}} \\
\Rightarrow a_{0}^{2}=\frac{G M_{\text {sphere }} \xi_{B}}{r_{B} \mu\left(\xi_{B}\right)},
\end{gathered}
$$

where we define

$\mu\left(\xi_{B}\right)=\left(\xi^{2} \frac{\mathrm{d} \psi}{\mathrm{d} \xi}\right)_{\xi_{B}}$.

The functions $\psi(\xi)$ and $\mu(\xi)$ are tabulated by integrating the isothermal Lane-Emden equation. The temperature within the sphere, $T_{\text {cld }}$, is calculated using Eqs. (1) and (4).

Particles within the sphere are randomly assembled using three random-number generators, each producing a randomnumber in the range $(0,1)$. A random-number $\mathcal{R}_{r}$ is chosen such that

$$
\begin{aligned}
& \frac{M(r)}{M_{\text {sphere }}}=\frac{\mu(\xi)}{\mu\left(\xi_{B}\right)} \equiv \mathcal{R}_{r} \\
& \Rightarrow \mu(\xi)=\mu\left(\xi_{B}\right) \mathcal{R}_{r} .
\end{aligned}
$$

Knowing the RHS of Eq. (5), the corresponding dimensionless radius $\xi$ can be obtained from the Lane-Emden table. The radial distance $r$ of a particle within the sphere is then given by $r=\mathcal{R}_{r} \xi$. The probability that a particle will lie in an infinitesimal interval of the poloidal angle $(\theta)$ and $(\theta+\mathrm{d} \theta)$ is then

$p_{\theta} \mathrm{d} \theta=\frac{\sin \theta}{2} \mathrm{~d} \theta$.

The total probability over the range of the angle $\theta$ is

$P(\theta)=\int_{\theta^{\prime}=0}^{\theta^{\prime}=\theta} \frac{\sin \theta^{\prime}}{2} \mathrm{~d} \theta^{\prime}=\frac{1-\cos \theta}{2} \equiv \mathcal{R}_{\theta}$,

where $\mathcal{R}_{\theta}$ is the second random number. Thus,

$\cos \theta=1-2 \mathcal{R}_{\theta}$, and finally the azimuthal angle, $\phi$, of a particle is fixed as, $\phi=2 \pi \mathcal{R}_{\phi}$, where $\mathcal{R}_{\phi}$ is a third random number. The $(r, \theta, \phi)$ coordinates are then converted to their cartesian form in the usual way.

The density of this sphere is

$\rho\left(r_{B}\right)=\frac{a_{0}^{6}}{4 \pi G^{3} M_{\text {sphere }}^{2}}\left(\xi^{2} \frac{\mathrm{d} \psi}{\mathrm{d} \xi}\right)_{\xi=\xi_{B}}^{2} \cdot \mathrm{e}^{-\psi\left(\xi_{B}\right)}$,

and $\xi_{B}=3$, by choice. However, such a sphere in isolation will simply diffuse away, and therefore needs to be spatially confined with a finite external pressure, $P_{\text {ext }}$, acting on its boundary. The magnitude of this pressure is

$P_{\text {ext }}=\frac{a_{0}^{8}}{4 \pi G^{3} M_{\text {sphere }}^{2}} \cdot \mu\left(\xi_{B}\right) \mathrm{e}^{-\psi\left(\xi_{B}\right)}$,

where all symbols have their usual meanings (Chandrasekhar 1939).

We simulate the warm external intercloud medium (ICM) by assembling particles in an envelope of finite thickness $\left(\delta \cdot h_{B}\right)$. Here $h_{B}$ is the average smoothing length of a single SPH particle and $\delta$, a non-zero integer defining the thickness of the envelope. Particles in this envelope interact with the gas particles within the cloud only by exerting hydrodynamic pressure of magnitude $P_{\text {ext }}$, defined by Eq. (8) above.

To prevent particles from diffusing across the cloud-ICM interface, care must be taken to avoid a gradient in particle number density. To ensure this, we first calculate the particle number density, $\hat{n}_{B}$, near the cloud edge and then evaluate the number of particles to be assembled in this envelope, $N_{\text {env }}$. The particle number density at the edge of the sphere is $\hat{n}_{B}=\frac{\hat{\rho}_{B}}{m_{i}} \equiv N_{\text {gas }} \hat{\rho}_{B}$, where $m_{i}$, the mass of the $i$ th particle in our test sphere is $m_{i}=\frac{1}{N_{\text {gas }}}$ and the volume of the ICM envelope is $\frac{4 \pi\left[\left(1+\delta \cdot h_{B}\right)^{3}-1\right]}{3}$, for a sphere of unit mass. The number of particles to be assembled in the envelope, $N_{\text {env }}$, is then the product of this volume with the particle number density, $\hat{n}_{B}$. The density at the cloud edge in its dimensionless form, $\hat{\rho}_{B}$, can be obtained by trivially manipulating Eq. (7). Thus we have,

$\hat{\rho}_{B}=\frac{\xi_{B}^{3} \mathrm{e}^{-\psi\left(\xi_{B}\right)}}{4 \pi \mu\left(\xi_{B}\right)}$.

The radial coordinate, $r$, of a particle in the envelope is fixed by choosing a random number, $\mathcal{R}_{r}$, such that the volume interior to this radius is proportional to the volume in the envelope, i.e.,

$\mathcal{R}_{r}=\frac{\left(r^{3}-1\right)}{\left(\left(1+\delta \cdot h_{B}\right)^{3}-1\right)}$.

The polar angles $(\theta, \phi)$ of individual particles are determined as before. The polar coordinates of particles are then converted to their cartesian form. With this, the assembly of the sphere envelope system is complete.

To test this scheme of assembling particles, we assembled 1500 particles within the sphere $\left(N_{\text {gas }}\right)$ and with a choice of $\delta$ $=5, N_{\text {env }}=6159$. This assembly was then placed in a periodic box and allowed to evolve for a fraction of the sound-crossing time. The periodic box is simply meant for ghosting particles i.e. particles leaving from one face of the cube re-enter from the opposite face. Figure 1 shows the SPH density of a truncated isothermal sphere, after about a half sound-crossing time, and it seems to be in reasonable agreement with the expected density profile, marked by the dashed continuous curve. 


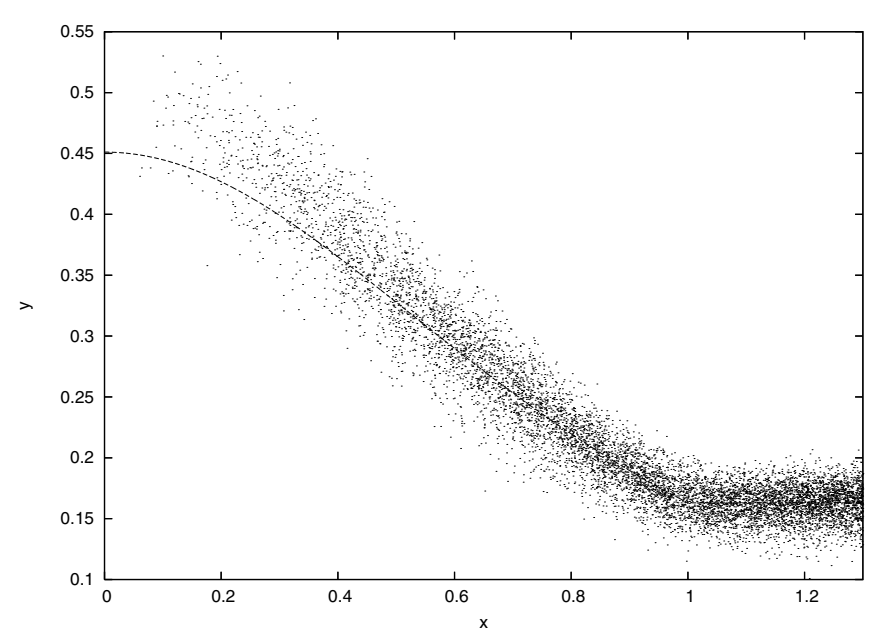

Fig. 1. This figure shows the SPH density, $\rho_{\mathrm{SPH}}$, of the truncated isothermal sphere $\left(\xi_{B}=3\right)$. The dashed continuous line is the expected density profile, $\rho\left(r_{B}\right)$, for this sphere. $\left(x \equiv \xi, y \equiv \rho_{\mathrm{SPH}}\right), N_{\mathrm{gas}}=1500$.

The box of the ICM particles is set up in a manner similar to the envelope of $P_{\text {ext }}$ particles for the truncated isothermal sphere, described above. The dimensions of the box are set so that each sphere has an envelope of thickness $3 h_{B}$. Figure 2 below shows a schematic diagram of the initial set up. It shows the precollision MCs barely touching each other in the plane of collision. Circles drawn with continuous lines represent the precollision clouds. Each circle is circumscribed by respective dashed circles that show the extent of the ICM envelope for each cloud

Since we opted to use physically identical clouds, each one of them has the same mass, $M_{\text {cld }}$, radius, $R_{\text {cld }}$, and an envelope of the ICM particles of thickness $\delta \cdot h_{B}$. The length of the ICM box along the axis of collision (the $x$ axis in Fig. 2) is

$x=4\left(R+\delta \cdot h_{B}\right)$,

while that along the $y$ and $z$ axes is

$y=z=2\left(R+\delta \cdot h_{B}\right)$,

and $\delta=3$ in the present case. A shortcoming of the SPH code used is that it requires a cubic periodic box. Each side of the cubic box therefore has length $x$ pc.

Let $N_{\text {gas1 }}$ and $N_{\text {gas2 }}$ be the number of gas particles in each cloud (provided externally). The number of particles to be assembled in the ICM envelope of respective spheres is $N_{\text {env } 1}$ and $N_{\text {env2 }}$. The region marked by stripes in Fig. 2 is the one occupied by the ICM particles, the volume of which can be trivially calculated. The number of particles to be assembled in this region can then be calculated using this volume and $\hat{n}_{B}$. The total number of particles in the box is simply the sum of $N_{\text {gas } 1}, N_{\text {gas } 2}, N_{\text {env1 }}$, $N_{\text {env2 }}$ and the number of ICM particles in the striped region of the box.

Particles in this box were assembled on a HCP lattice. Particles within the desired cloud radius are then scooped out and replaced with corresponding gas particles; the particles in the second cloud are also assembled in a similar fashion. This concludes the process of setting up the ICs.

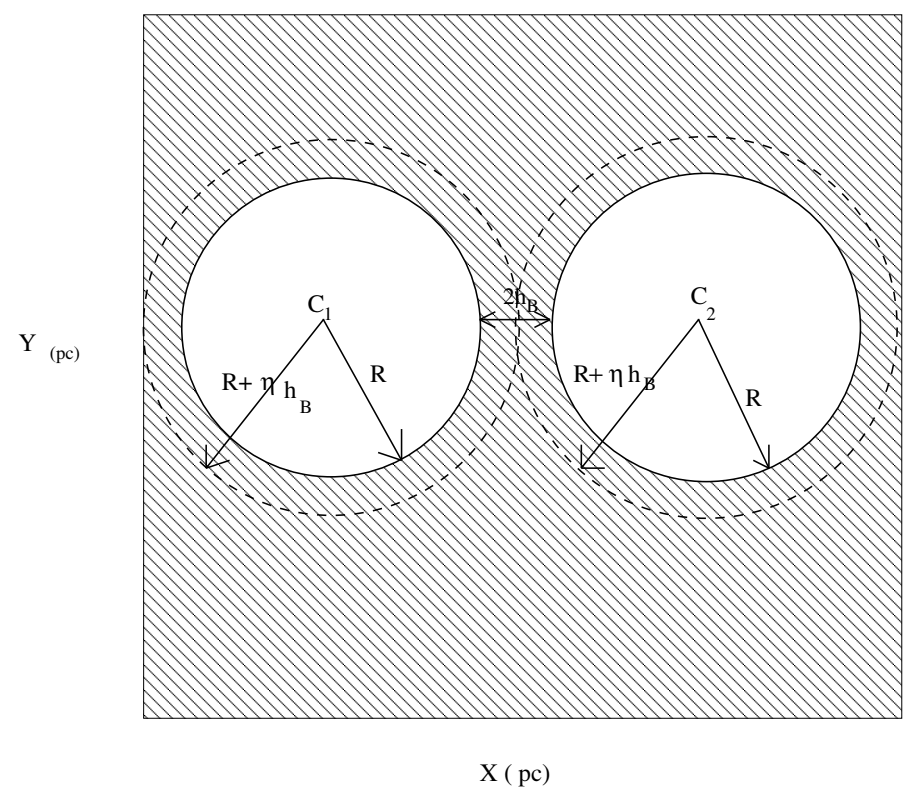

Fig. 2. Schematic diagram of the initial set up of colliding clouds. See text above for description.

\subsection{Equation of state (EOS)}

In the simulations discussed here, we used a slightly modified barytropic EOS given by

$$
\begin{aligned}
& \frac{P}{\rho}=\left(k_{\mathrm{B}} / \bar{m}\right) \\
& \times\left\{\begin{array}{l}
\left(\frac{T_{\text {cld }}}{\mathrm{K}}\right) ; \rho \leq 10^{-21} \mathrm{~g} \mathrm{~cm}^{-3} \\
\left(\frac{\gamma T_{\mathrm{cld}}}{\mathrm{K}}\right)\left(\frac{\rho}{5 \times 10^{-21} \mathrm{~g} \mathrm{~cm}^{-3}}\right)^{\gamma-1} ; 10^{-21} \mathrm{~g} \mathrm{~cm}^{-3}<\rho \leq \\
5 \times 10^{-21} \mathrm{~g} \mathrm{~cm}^{-3} \\
\left(\frac{T_{\mathrm{ps}}}{\mathrm{K}}\right) ; 5 \times 10^{-21} \mathrm{~g} \mathrm{~cm}^{-3}<\rho \leq 10^{-18} \mathrm{~g} \mathrm{~cm}^{-3} \\
\left(\frac{T_{\mathrm{cld}}}{\mathrm{K}}\right)\left[1+\gamma\left(\frac{\rho}{10^{-14} \mathrm{~g} \mathrm{~cm}^{-3}}\right)^{\gamma-1}\right] ; \rho>10^{-18} \mathrm{~g} \mathrm{~cm}^{-3}
\end{array}\right.
\end{aligned}
$$

Here, $T_{\text {cld }}$, is the pre-collision cloud temperature, $T_{\mathrm{ps}}$ the post shock temperature, $k_{\mathrm{B}}$ the Boltzmann constant, $\bar{m}$ the mean mass of a hydrogen molecule, and $\gamma$ the adiabatic constant which, in the present case is $\frac{5}{3}$. This comes from our assumption of monatomic gas within individual clouds.

The density in the thin dissipative region downstream of a strong adiabatic shock is at most four times the preshock density, a direct consequence of the Rankine-Hugoniot jump condition. Gas behind a strong shock radiates efficiently, and the shock may thus be treated as cool and approximately isothermal. In the next few lines we attempt to justify our assumption that the post-collision gas slab in the models tested here is approximately isothermal. That would be a rather straightforward task in case a post-shock cooling scheme were employed. Nonetheless, one may summon for the purpose the average post shock density, temperature and a model for radiative cooling proposed by Semadeni et al. (2007). The temperature jump condition for models 2 and 3 implies a post-shock temperature, $T_{\mathrm{ps}}$, of order $6000 \mathrm{~K}$ and $11000 \mathrm{~K}$, respectively, while that for model 4 is the same as in model 2 . The cooling rate suggested by the authors would then correspond to a cooling time, $\tau_{\text {cool }}$, of only a few thousand years. In that case, the cooling length, $l_{\text {cool }}$ $\left(l_{\text {cool }} \sim \tau_{\text {cool }} a_{0}\right)$, over which the shocked gas downstream a shock 
Table 1. List of simulations performed.

\begin{tabular}{|c|c|c|c|c|c|}
\hline $\begin{array}{l}\text { Serial } \\
\text { No. }\end{array}$ & $\begin{array}{l}\text { Experimental } \\
\text { details }\end{array}$ & $\begin{array}{l}\text { Pre-collision } \\
\text { Mach number }(\mathcal{M})\end{array}$ & $P_{\mathrm{ext}}^{*}$ & $\begin{array}{r}\text { Number of } \\
\text { particles }\end{array}$ & Head-on* \\
\hline 1 & $\begin{array}{l}M_{\mathrm{cld} 1}=M_{\mathrm{cld} 2}=100 M_{\odot} \\
R_{\mathrm{cld} 1}=R_{\mathrm{cld} 2}=1 \mathrm{pc}, T_{\mathrm{cld}}=84 \mathrm{~K}\end{array}$ & 35 & Yes & $\begin{array}{r}N_{\text {tot }}=34893 \\
N_{\text {gas }}=3516 \\
\end{array}$ & Yes \\
\hline 2 & $\begin{array}{l}M_{\mathrm{cld} 1}=M_{\mathrm{cld} 2}=50 M_{\odot} \\
R_{\mathrm{cld} 1}=R_{\mathrm{cld} 2}=0.8 \mathrm{pc}, T_{\mathrm{cld}}=54 \mathrm{~K}\end{array}$ & 25 & Yes & $\begin{array}{r}N_{\text {tot }}=129427 \\
N_{\text {gas }}=16000 \\
\end{array}$ & Yes \\
\hline 3 & $\begin{array}{l}M_{\mathrm{cld} 1}=M_{\mathrm{cld} 2}=400 M_{\odot} \\
R_{\mathrm{cld} 1}=R_{\mathrm{cld} 2}=2.0 \mathrm{pc}, T_{\mathrm{cld}}=170 \mathrm{~K}\end{array}$ & 25 & No & $N_{\text {gas }}=120000$ & Yes \\
\hline 4 & $\begin{array}{l}M_{\mathrm{cld} 1}=M_{\mathrm{cld} 2}=50 M_{\odot} \\
R_{\mathrm{cld} 1}=R_{\mathrm{cld} 2}=0.8 \mathrm{pc}, T_{\mathrm{cld}}=54 \mathrm{~K}\end{array}$ & 25 & No & $N_{\mathrm{gas}}=40000$ & $\begin{array}{c}\text { No } \\
b=0.2 \mathrm{pc}\end{array}$ \\
\hline
\end{tabular}

* Clouds confined by external pressure, $P_{\text {ext }}$, exerted by the ICM particles.

$\star$ Indicates if the clouds were allowed to collide head-on/off-centre; $b$ is the impact parameter of collision.

cools to the original preshock temperature, would only be a tiny fraction of the observed slab thickness in the simulations performed. A short cooling length and cooling time (compared to the sound crossing time) presage the assumption of isothermality behind a shock.

To conclude the argument, let us additionally calculate $\tau_{\text {cool }}$ using the relation

$\tau_{\text {cool }} \sim \frac{2.4 \times 10^{5}}{\bar{n}_{\mathrm{H}}} \mathrm{yrs}$.

Here $\bar{n}_{\mathrm{H}}$ is the post-shock number density of atomic hydrogen (Spitzer 1978). Even by conservative estimates if we were to assume a compression factor of 4 as in a strong adiabatic shock, the post-shock density would be of order $10^{3} \mathrm{~cm}^{-3}$, so that Eq. (11) implies $\tau_{\text {cool }}$ is only about a few hundred years, much shorter than the local sound crossing time.

For the models tested here, the post shock density is $\sim 10^{-21} \mathrm{~g} \mathrm{~cm}^{-3}$. Thus, for gas density below this threshold, we adopt an isothermal EOS and maintain the gas at the initial cloud temperature, $T_{\text {cld }}$. Following the cloud collision for densities above this threshold and less than $5 \times 10^{-21} \mathrm{~g} \mathrm{~cm}^{-3}$, we adopt an adiabatic EOS to model the shock. The gas is then held at a post shock temperature, $T_{\mathrm{ps}}$. When the shocked gas becomes sufficiently dense $\left(\sim \mathcal{M}^{2} \rho_{\text {cld }} \sim 10^{-18} \mathrm{~g} \mathrm{~cm}^{-3}\right)$, its temperature is brought down to that set in the initial, pre-collision clouds, $T_{\text {cld }}$. The cold, approximately isothermal gas slab is then so maintained up to a density of $10^{-14} \mathrm{~g} \mathrm{~cm}^{-3}$. Above this threshold, the EOS flips from being isothermal to adiabatic. This final phase is meant to follow putative star-forming clumps in the shocked slab.

\subsection{Models tested}

The cloud collision experiments presented here are listed in Table 1 above. The table provides the relevant physical details of an experiment. We arbitrarily chose the precollision Mach number, $\mathcal{M}$, and the cloud mass, $M_{\text {cld }}$. The corresponding cloud radius, $R_{\text {cld }}$, was determined using the Larson's scaling relation between mass and radius,

$R_{\text {cld }}(\mathrm{pc})=0.1(\mathrm{pc})\left(\frac{M_{\text {cld }}}{M_{\odot}}\right)^{0.5}$

(Larson 1981).

The fate of the post-collision slab largely depends on the initial conditions. We observed contrasting features in the evolution of the shocked slab in models 1, 2, and 3. While model 1 is under resolved and just a crude test case, the last two are much better resolved, with more gas particles.

\subsubsection{Model 1}

In this case the clouds collide head-on at a high supersonic speed. Post-collision, the clouds coalesce to form a shockcompressed gas slab, which evolves through the compression and expansion phases described below. Artificial viscosity dissipates the kinetic energy of colliding clouds and heats up the shocked slab, whose temperature is denoted as $T_{\mathrm{ps}}$. The gas slab in this case does not become Jeans unstable, presumably for the following two reasons: (i) insufficient resolution due to fewer particles and (ii) the post-shock density that is less than is required to support the Jeans instability.

Consequently, the slab simply expands along the collision axis. Also, there is no evidence of any other instability in the shocked slab. The fate of such a collision may be envisaged as a diffuse cloud, a part of the ICM. To test this outcome, we performed a similar experiment in the next case.

\subsubsection{Model 2}

Although the precollision cloud speed in this case is somewhat lower than in the previous case, individual clouds are still highly supersonic. Column density plots in Fig. 3 show a time sequence of the colliding clouds in this model. Formation of the contact layer can be seen in the picture corresponding to $t=0.01 \mathrm{Myr}$. The slab then develops a kink on it (the bending of the slab), which then rapidly amplifies, as can be seen in the snapshots corresponding to $t=0.03 \mathrm{Myr}, t=0.07 \mathrm{Myr}$ and $t=0.085 \mathrm{Myr}$. Although the slab layers are sheared, the gravitational instability dominates in this case. The shocked slab fragments and forms a network of filaments. Naively speaking, the slab develops a number of holes in it and gas ablates in to these filaments.

These filaments subsequently become gravitationally unstable and produce dense clumps in them. The face-on picture of the fragmented slab in Fig. 4 shows filamentary network and the clumps within them. The gravitational instability grows rapidly, and the slab fragments on a timescale much smaller than $t_{\mathrm{cr}}$, the cloud crushing time defined in Sect. 4.2 below. The criterion for dominance of the gravitational instability over shearing instabilities is

$$
\bar{n} \gtrsim 4.7 \times 10^{2}\left(\frac{\eta}{\mathrm{pc}}\right)\left(\frac{\lambda_{\mathrm{NTSI}}}{\mathrm{pc}}\right)^{-3}\left(\frac{T}{\mathrm{~K}}\right) \mathrm{cm}^{-3},
$$

where $\bar{n}$ is the gas number density in the slab, $\eta$ the amplitude of perturbation, and $\lambda_{\mathrm{NTSI}}$ the length of an unstable mode (Heitsch, Hartmann \& Burkert 2008).

From the simulation we find that $\eta \sim 0.02 \mathrm{pc}$ and $\lambda_{\mathrm{NTSI}} \sim$ $0.01 \mathrm{pc}$, so that Eq. (13) yields $\bar{n} \sim 10^{5} \mathrm{~cm}^{-3}$. In the present 

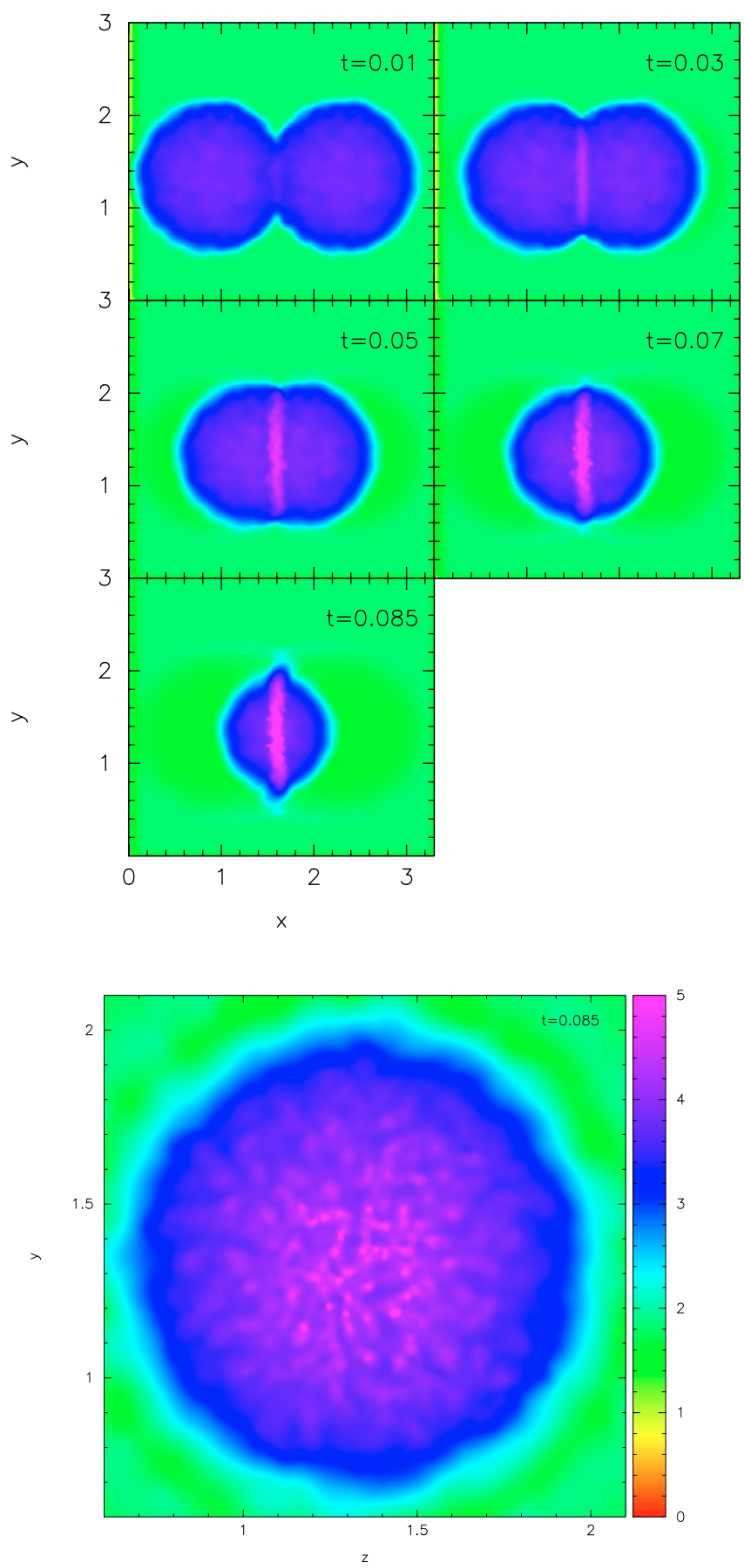

Fig. 4. A column density plot of the fragmented slab in model 2. We can see a network of filaments with several clumps in them, marked as bright pink blobs. The clumps are actually sink particles. Time measured in Myr.

case the post-shock density is $\sim 10^{-18} \mathrm{~g} \mathrm{~cm}^{-3}$, so that the corresponding number density is $\sim 10^{6} \mathrm{~cm}^{-3}$, which is nearly an order of magnitude higher than the calculated $\bar{n}$. The shocked slab is therefore susceptible to gravitational instability. This outcome, however, contrasts with that of the previous case where the gas slab evolved into a diffuse cloud.

\subsubsection{Model 3}

This model is similar to the previous two except that the precollision clouds here are unconfined; i.e., there are no ICM particles.

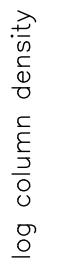

Fig. 3. A column density plot showing a time (in Myr) sequence of the colliding clouds in model 2. The collision produces a shocked slab that develops bending modes, and kinks in the slab are visible in the plots in the second row. Some material also squirts out, jets, from the top and bottom ends of the slab $(t=0.85 \mathrm{Myr})$.

Figure 5 below shows a time sequence of column density plots of the colliding clouds through to the development of the NTSI in the shocked slab. The initial behaviour of the post-collision gas slab is similar to the previous case, the NTSI eventually dominates the dynamical evolution of the slab. The gravitational instability thus seems to be suppressed. Another instability to which the slab is susceptible is the Kelvin-Helmholtz (KH) instability, the distinct feature of which is the formation of vortices. Material from colliding clouds streams in from either side of the contact layer and smears laterally after striking it. Obviously, the gas elements within the slab are in a turbulent state, and artificial viscosity is responsible for shearing interaction between them.

Sure, SPH in its simplest form is unable to intricately resolve the vortex rolls associated with the KH instability, but it can at least represent the underlying physical process, of layers mixing. This produces a non-uniform velocity field in the plane of the slab is evident from the plot in the top panel of Fig. 6. This is a 3-d plot showing the velocity field surface in the plane of the slab. The number of secondary peaks on this surface correspond to the localised circular velocity patterns in the slab, obvious features of the $\mathrm{KH}$ instability. In the bottom panel, the velocity field has been overlayed on the column density plot of the slab as seen by an observer stationed in a direction orthogonal to the plane of collision. From this plot we can see that the slab develops kinks, and the bending modes are more readily visible in its central plane.

With the help of first-order perturbative analysis, it can also be shown that fluid surfaces in shearing contact develop surface gravity waves that give them a rippled appearance also called, the breathing modes of the fluid surface. This situation is analogous to the formation of ripples on the surface of water bodies (Raichaudhari 1999; Shore 2007). Figure 7 below is a column density plot showing a face-on view of the shocked slab. Density contours have been overlayed to facilitate identification 


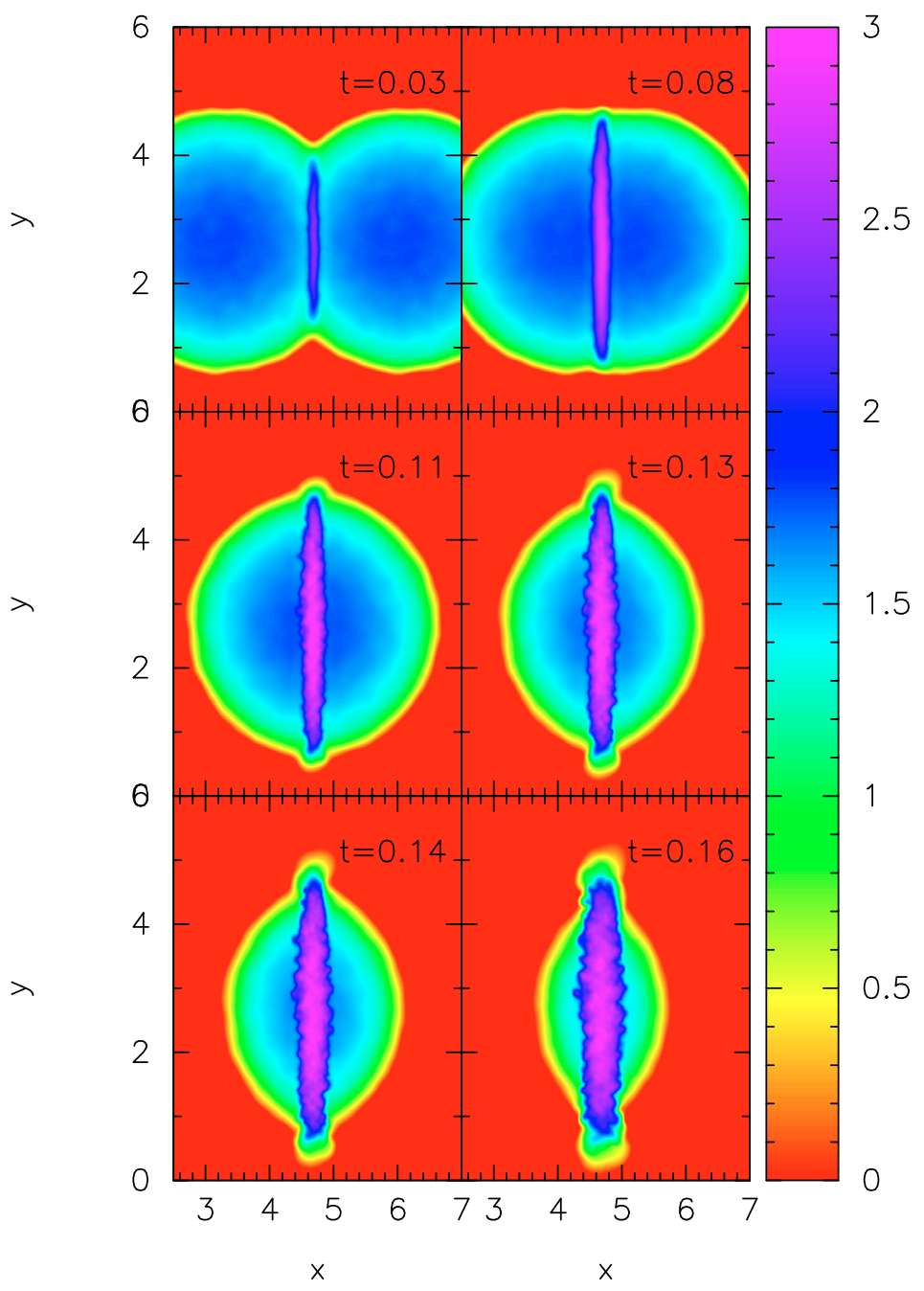

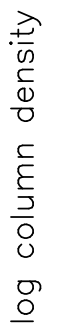

Fig. 5. A time (measured in Myr) sequence of the column density plots in model 3. Formation of the shocked slab can be seen in the snapshot corresponding to $t=$ 0.03 Myr. The NTSI then dominates the slab, as can be seen in the snapshots in the second and third rows, after which the slab soon becomes bloated $(t=0.16 \mathrm{Myr})$. of structure within the slab. We can see a dense network of a filamentary structure, and a clump $\left(\rho \sim 10^{-18} \mathrm{~g} \mathrm{~cm}^{-3}\right)$ formation is visible in regions where filaments intersect, however, this structure suffers from tidal disruption due to strong shearing motion between slab layers. Klessen \& Burkert (2000) and Vietri et al. (1997), for instance, make similar observations about ablation of clumps in their models of turbulent GMC interiors.

Since we started with unconfined clouds, the shocks confining the gas slab move outwards (essentially vacuum in the simulation) and the slab expands and then collapses laterally. See the plot of particle positions corresponding to $t=0.50 \mathrm{Myr}$ in Fig. 12. The thermal pressure within the slab is insufficient for arresting the collapse, which proceeds towards the formation of a filament along the collision axis. Gravitational clustering may then commence in this filament. However, we have not followed the simulation to that point because of constraints on resources. We note that grid codes simulating colliding clouds suggest that the expansion followed by the collapse phase is dominated by the Rayleigh-Taylor (RT) and KH instabilities (c.f. Miniati et al. 1997). However, there is no evidence of the RT instability in this model, although vortex formation in the slab suggests the latter.

\subsubsection{Model 4}

In this case unconfined clouds collide at a finite impact parameter, $b=\frac{R_{\text {cld }}}{4}$, with other physical parameters, the same as those in model 2. The supersonic cloud collision results in an oblique shock compressed slab. Unlike the planar slabs formed in a headon collision, the oblique shocked slab has three distinct regions: the central planar region and the two arched extensions at the top and bottom ends of the slab. See snapshots in the panels of Fig. 8. These extended features stem from the initial shock front effectively blocking the fluid flow from either side in the central regions, while the top end where the fluid flows from the right to left has higher transverse velocity than that in the central regions. For the same reason there is also an extension at the lower end of the slab, except that the fluid at this end is flowing from the left to right.

Like the former cases, the shocked slab in this case is also internally stressed and evolves into a filament aligned with the collision axis. Like the previous two cases, the shocked slab in this case also quickly develops structure. Elongated density structures and blobs can be seen in Fig. 9, which is a column density plot of the slab as seen face-on.

\section{Discussion}

Collision between clouds is characterised by three phases.

a) The compression phase, during which first a contact layer forms post-collision and shock waves propagate in individual clouds. The slab is confined by ram pressure on either side, which strongly compresses the gas within the slab. The thickness of the slab depends on the effectiveness of the artificial viscosity. Additionally, it could also depend on the 


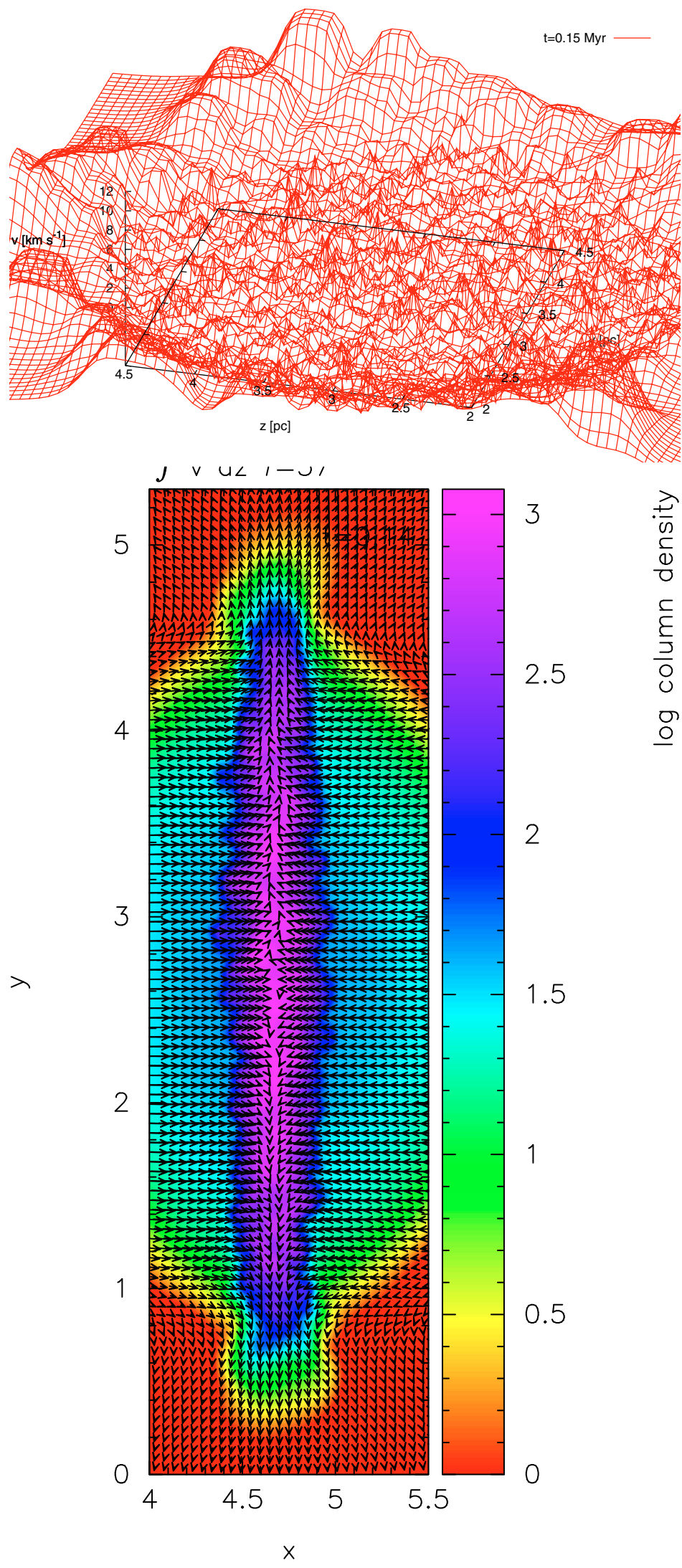

efficiency of radiative cooling, but this is not the case in the present work as we use a plain EOS.

(b) The next phase is that of expansion. During this phase, the shocks confining the slab propagate outward in the ICM,
Fig. 6. Top panel: a plot of the velocity field $(t=0.14 \mathrm{Myr})$ in the plane of the slab in model 3 . It is evident that the velocity field within the slab is highly uneven and the spikes in it suggest formation of vortices in the slab. Bottom panel: velocity field in the plane of collision overlayed on column density plot of the slab $(t=0.14 \mathrm{Myr})$ in model 3. The kinks in the slab along with the jets are evident from this plot. Velocity is measured in $\mathrm{km} \mathrm{s}^{-1}$.

and a rarefaction wave propagates back into the slab. This creates a central low-pressure and low-density region in the slab. During this phase, some material squirts out from the top and the bottom of the slab. This is also the phase during 


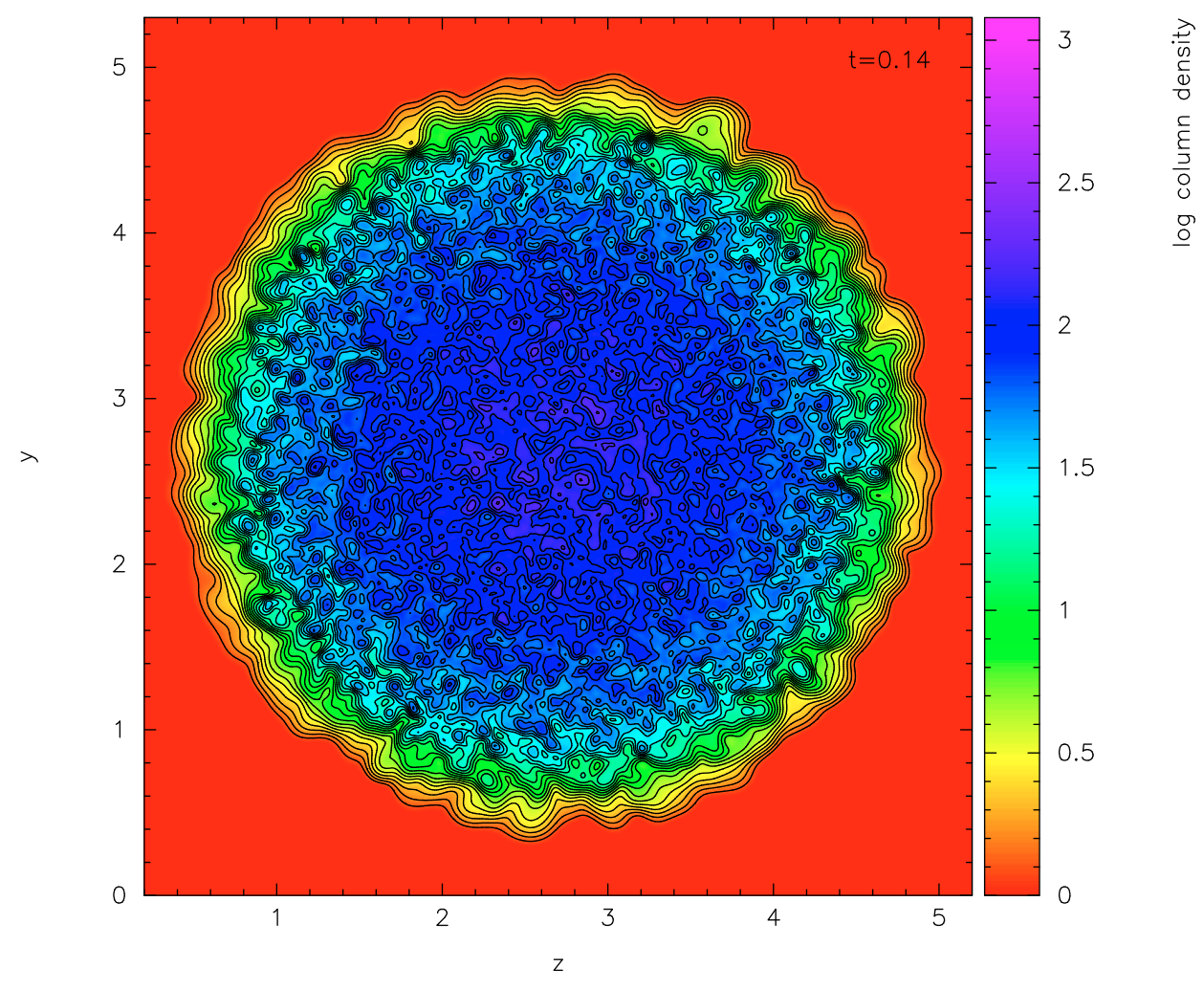

Fig. 7. A column density plot of the shocked slab in model 3, as seen in the plane orthogonal to the plane of collision. Density contours overlayed on it show several elongated features and dense clumps within them. Time measured in Myr.

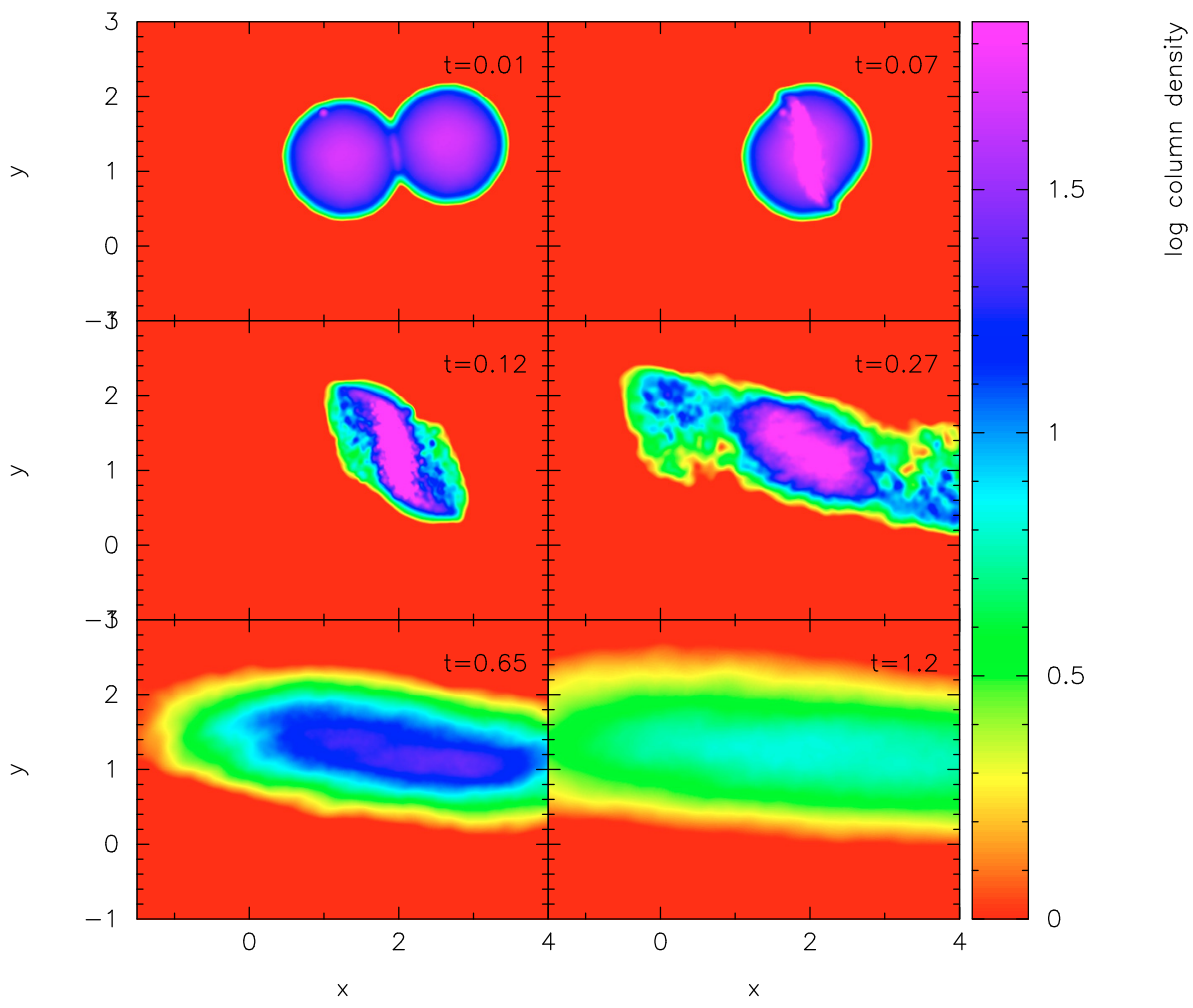

Fig. 8. A time (measured in Myr) sequence of column density plots in model 4 . The off-centre cloud collision leads to the formation of an oblique shocked slab as can be seen in the pictures of the top row. In the second row we can see that the slab tumbles about the $z$ axis and breaks into three parts, with the two outer lobes moving away from the central region. In the bottom row we see that the central region evolves into a filament aligned with the collision axis. which the slab may become susceptible to the NTSI and the $\mathrm{KH}$ instability due to lateral motion of the gas within the slab at supersonic speed (relative to the local sound speed in the slab).

(c) The final phase is that of lateral collapse. The slab expansion is halted by the ICM, by which time pressure within the slab becomes much lower than the external pressure. Consequently the expanding slab material is driven towards the central low-density region.

(d) If the externally confining pressure is too weak, the final phase could be different and the expanding slab may simply form a diffuse cloud, amalgamated with the ICM. 


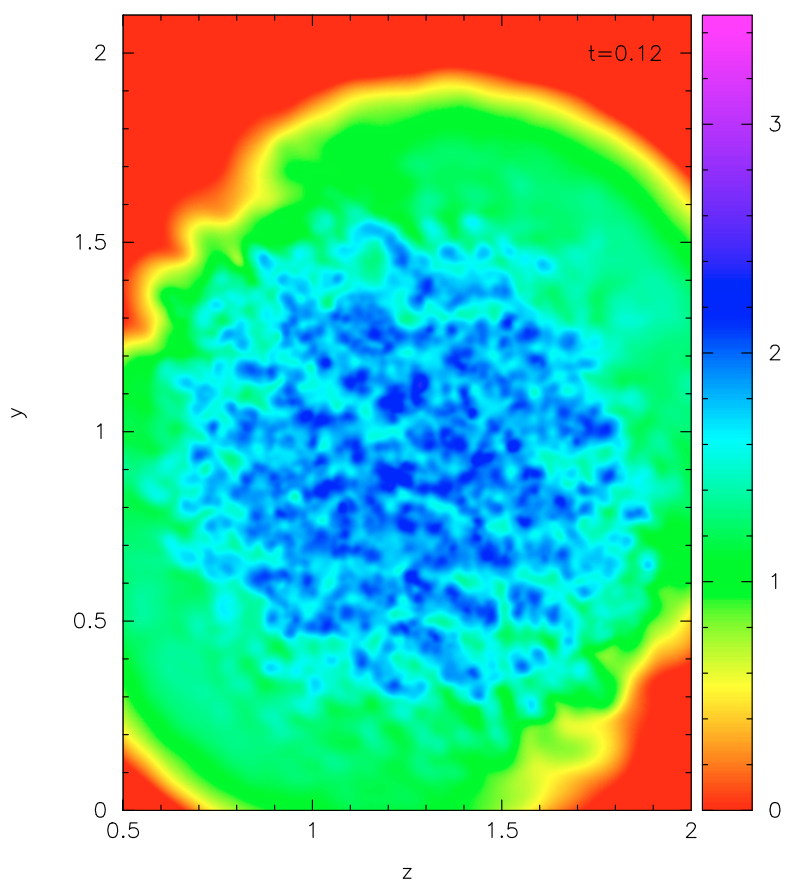

Fig. 9. A column density plot of the shocked slab in model 4 shortly after its formation. Structure within the slab is evident from the plot along with dense clumps, at the intersection of filaments. Time measured in Myr.

\subsection{Instabilities in a shocked slab}

The problem under investigation here is that of the stability of a viscous fluid slab confined by accretion shocks on its either side. Growth of instabilities in a cold, isothermal slab has been extensively studied in the past. Linear perturbative analyses have shown that the isothermal slab has several unstable modes; see for instance, Ledoux (1951), Chandrasekhar (1953), Mestel (1965), and Simon (1965). However, these investigations assumed slabs with no external forces acting on them.

A shocked slab, on the other hand, shows propensity for a variety of instabilities. However, the present investigation is limited to the gravitational and shearing instabilities since other relevant physical details have been avoided in this work. The final state of the shocked slab depends on the relative dominance of either of the two instabilities. The shearing instabilities are mainly of two varieties: (a) the non linear thin shell instability (NTSI) (b) the Kelvin-Helmholtz (KH) instability. The condition for the dominance of the gravitational instability over the shearing instabilities, especially the NTSI, is given by Eq. (13) above.

\subsubsection{The NTSI}

Growth of instabilities in shocked slabs was first studied by Vishniac (1983), by invoking full shock conditions, who suggested the existence of bending modes of a shocked slab. His linear analysis of the growth of these modes showed that the instability becomes overstable and led to disintegration of the slab. The resulting fragments suffered erosion due to the RT instability. Gravitational instabilities are suppressed in this regime. In the limit of a small bending angle, the linear analysis is sufficient for predicting the growth rate of the unstable mode. The linear theory, however, breaks down when velocities within the slab become comparable to the local sound speed and fluctuations in column density become of order unity.
This investigation was extended by Vishniac (1994) to the non linear regime of the bending and breathing modes. The analysis showed that an isothermal shocked slab of inviscid fluid became unstable due to non linear growth of the unstable modes. Since this instability commences in thin shells (i.e. between layers of the slab), it is called the thin shell instability. The instability grows rapidly and saturates, leading to turbulent mixing between layers of the slab. The slab eventually forms a sievelike structure and becomes bloated before finally disintegrating to form a filament aligned along the collision axis. Gravitational clustering can then occur in this filament. A similar observation has also been reported by Hueckstaedt (2003) in grid simulations of colliding fluid flows. According to Burkert \& Hartmann (2004), a cold, self-gravitating gas slab collapses on a timescale, $t_{\mathrm{c}}$, and

$t_{\mathrm{c}}=\left(\frac{R_{\mathrm{cld}}}{\pi G \Sigma_{\mathrm{cld}}}\right)^{\frac{1}{2}}$,

where $\Sigma_{\text {cld }}$ is the slab surface density. In case of model 3 for instance, $t_{\mathrm{c}}$ is about $1 \mathrm{Myr}$, and from Fig. 12 below, we can see that the gas slab has collapsed substantially by $t=0.5 \mathrm{Myr}$.

Although we start with unperturbed initial conditions, the perturbations that develop in the shocked slab are purely from white noise. However, the simulation has sufficiently high resolution to resolve the observed NTSI wavelength, $\lambda_{\text {NTSI. }}$ In SPH, the spatial extent of the smallest region resolved is of the order of the particle smoothing length, $h$. An SPH sphere of influence has volume $32 \pi h^{3} / 3$, so that for a shocked slab with $N_{\text {gas }}$ particles,

$h=\left(\frac{3 R_{\text {cld }}^{2} N_{\text {neibs }} \Delta x}{32 N_{\text {gas }}}\right)^{\frac{1}{3}}$

where $\Delta x$ is the shock thickness and remaining symbols have their usual meanings. For the chosen dimension of clouds, the smoothing length is at least an order of magnitude smaller than $\lambda_{\text {NTSI }}$. We therefore believe that the features of the instability observed in our simulations are physical.

As a shocked slab accretes material, small perturbations, i.e. the bending modes of the slab get amplified due to non linear transfer of momentum within the slab. Material streaming in the perturbed gas slab deposits a net positive momentum in the convex regions and a net negative momentum in the concave regions of the slab. Transfer of momentum within the slab amplifies its bending modes to scales greater than the thickness of the slab itself. It is essential to note that the pressure within the slab is thermal and isotropic, while the external one is ram pressure. This distinction is irrelevant in the unperturbed state.

However, once a bending mode of the slab is excited, there is a net imbalance in forces acting on the convex and concave regions of the slab leading to its amplification. In general, the accreted material converges in the convex regions of the slab and diverges in the concave regions. Slab layers undergo turbulent mixing due to the strong shear between them. If $\tau$ is the dynamic timescale of the shocked slab (i.e. the timescale on which physical properties of the slab evolve dynamically), turbulent mixing occurs when $\tau$ is greater than the time required for the generation of the next similar layer at a distance greater than the wavelength and with wavenumber $k$. If $L$ is the thickness of the slab and $t$ is its age, then

$\tau \gg \frac{t}{k L}$

is the condition under which turbulent mixing is suppressed (Vishniac 1994). 
If $\tau \sim t$, the turbulent mixing is weak and is only marginally effective in the post-shock slab. The turbulent mixing of slab layers gives rise to eddies on the scale of slab thickness which is an effective means of energy dissipation. The NTSI grows at a rate

$\tau^{-1} \sim a_{0} k\left(k x_{m}\right)^{1 / 2} \cdot C_{d}^{-1 / 2}$

(Vishniac 1994).

Here $x_{m}$ denotes the midplane of the slab and $C_{d}$ a constant of order unity. This constant describes the extent of dissipation resulting from mixing between slab layers. Klein \& Woods (1998) estimate $2 \lesssim C_{d} \lesssim 4$ and a rate of saturation $>a_{0} k(k L)^{1 / 2}$.

\subsection{Observations from simulations}

Clouds collide and first form a layer of contact, after which shock waves propagate into individual clouds. The cloud material streams into the layer and the timescale on which a cloud is destroyed is called the cloud-crushing time, $t_{\mathrm{cr}}$. Shock waves propagating in a cloud reach its far edge in this much time, and $t_{\text {cr }} \sim \frac{2 R_{\text {cld }}}{v_{\mathrm{s}}}$ (Klein \& Woods 1998). Here $v_{\mathrm{s}}$ is the velocity of the shock wave.

The density and pressure (thermal + ram) gradients respectively, have opposite signs across the shocked slab. The slab surfaces are shocked while pressure within the slab is thermal. Thus the pressure gradient across the slab surface has an opposite sign relative to the density gradient, which increases steeply. The shocks confining the slab apparently arrest the inflow of gas from the individual clouds. The colliding clouds are therefore held up.

This can be seen, for instance, in model 2 (Sect. 3.3.2). The column density plot corresponding to $t=0.085 \mathrm{Myr}$ (greater than $t_{\mathrm{cr}}$ ) in Fig. 3 above shows that the colliding clouds survive even beyond their crushing time, $t_{\mathrm{cr}}$. In fact at this epoch, the shocked slab has undergone gravitational fragmentation. Figure 4 shows the column density plot ( $t=0.085 \mathrm{Myr}$ ) of the fragmented slab. Filamentary structure within the slab is evident in this plot. The filaments then develop condensations and form a number of clumps in them.

The clumps are actually sink particles, and we remind our reader that sinks in the present work represent protostellar cores. Soon after its commencement, core formation spreads like an epidemic, and within a few thousand years, sixteen cores are formed in various filaments, whence the simulation was terminated. Cores accrete material channelled along their natal filaments.

Immediately after its formation the shocked slab develops kinks, and features of the NTSI are visible in it. The bending modes of the slab subsequently amplify, and it soon becomes bloated. This can seen in the snapshots corresponding to $t=0.03 \mathrm{Myr}, t=0.05 \mathrm{Myr}$ and $t=0.07 \mathrm{Myr}$ of Fig. 3 . Interestingly, there is a confluence of the gravitational instability and the NTSI, but the former dominates the latter and the condition for dominance of the gravitational instability is given by Eq. (13) above. Thus, we note that the gravitational instability can dominate the shocked slab even in presence of the NTSI, provided the slab is sufficiently dense.

Although we have seen evidence of clump formation in this model, only the self-gravitating clumps survive. Other smaller clumps either merge to form larger clumps or simply diffuse away. We therefore envisage this model as one leading to the formation of a filamentary structure, which may go on to spawn star formation. This is therefore an interesting case from the perspective of star formation. However, we do not observe any evidence of secondary fragmentation of cores in this model.

In model 3 we performed a similar experiment with higher resolution but no intercloud medium. The shocks confining the post-collision slab move outward (into vacuum) and thus permit expansion of the slab, as can be seen in the pressure-distance plots of Fig. 11. Distance in parsecs is marked on the $x$-axis and pressure, normalised relative to the initial pressure, has been marked along the $y$-axis. The plots in the top row $\left(t=0.48 t_{\mathrm{cr}}\right)$, correspond to the post-collision jump in density and pressure, respectively. The plot of pressure shows the formation of shocks at the slab surfaces, while that within the slab is thermal and uniform.

The pressure and density gradients have opposite signs across the slab surfaces, making it unstable. Unlike Hunter et al. (1986), we are, however, disinclined to term this situation as Rayleigh-Taylor (RT) unstable. The outwardly propagated and weakened shocks can be seen in the plots corresponding to $t=11.4 t_{\mathrm{cr}}$. During this phase, the central density and pressure within the slab falls, as can be seen in the plots in the second and third rows of this figure. Spikes in the density distribution indicate clump formation, which obviously do not survive. The slab continues to expand and flattens laterally. Eventually it becomes elongated with a large aspect ratio, and its long axis lies in the plane of collision. Figure 12 shows a plot of particle positions in the plane of collision.

As the shocked slab evolves, a few breakaway blobs are visible near its surface. These can be seen in the column density snapshot corresponding to $t=0.16 \mathrm{Myr}$ in Fig. 5. This perhaps is due to the inability of SPH in its simplest form to model the $\mathrm{KH}$ instability, apparently due to formation of artificial boundaries between regions having steep density contrast (Agertz et al. 2007). This problem can be fixed by including some additional correction terms to account for thermal energy dissipation across these boundaries (Price 2007 II). Our code does not include these corrections; however, this shortcoming does not seem to have any adverse bearing on the results of the simulations. Neither is it not our aim here, to model the $\mathrm{KH}$ instabilities in their minute details.

Similar breakaway blobs, having much lower densities (at least by two orders of magnitude), as compared to those observed in our simulations, have been reported by Heitsch et al. (2008). They interpret this observation as evidence of core formation. While these authors began with perturbations on lateral faces of colliding fluid streams and grid codes, apparently, being able to better handle density contrasts, it is plausible that their observation of core formation is physical; however, we prefer to discount the blobs as numerical artifacts despite they having densities about an order of magnitude higher than that of the slab.

Further as in the previous case, the shocked slab in this case also shows features of the NTSI. See column density plots corresponding to $t=0.11$ through to $0.16 \mathrm{Myr}$, in Fig. 5 above. These pictures show that the kinks (bending modes) in the slab grow rapidly, and soon it becomes bloated $(t=0.16 \mathrm{Myr})$. In the bottom panel of Fig. 6, the local velocity field has been overlayed on the column density plot of the shocked slab $(t=0.14 \mathrm{Myr})$, by which time features of the NTSI have developed well. This picture shows amplified kinks in the slab. By measuring the amplitude of the perturbation and the slab thickness, we calculated the growth rate of the NTSI as

$\tau^{-1} \sim(0.56)(k L)^{0.78}$ 


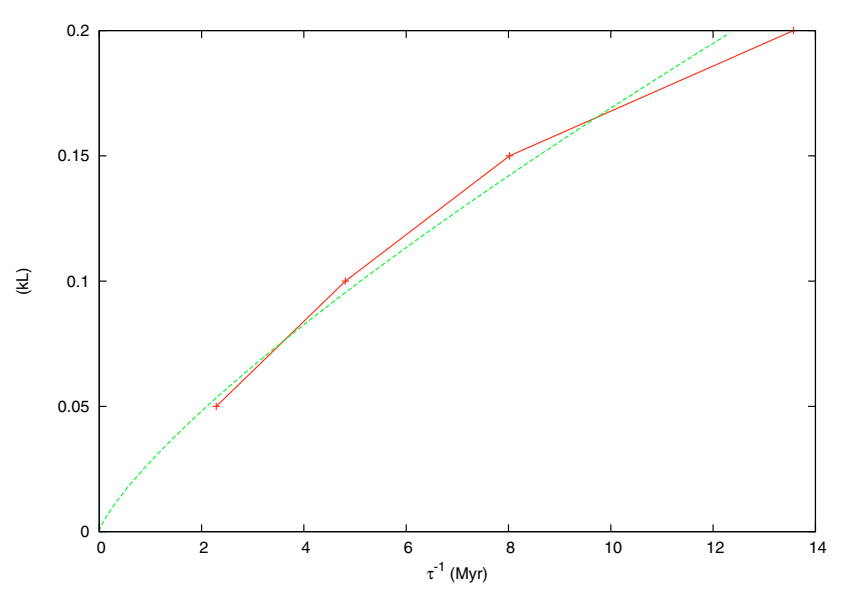

Fig. 10. The growth rate of the NTSI in model 3. The red curve corresponds to the actual growth rate of NTSI in the simulation, while the green curve is a power-law fit (Eq. (16)) to the observed growth rate. Time on the $x$ axis is measured in units of the cloud crushing time $t_{\mathrm{cr}}$.

where $k$ is the wavenumber of the unstable mode and $k=\frac{2 \pi}{\lambda_{\text {NTSI }}}$, $L$ is the thickness of the slab. Figure 10 shows the growth rate of the NTSI.

The observed growth rate of the NTSI in this simulation agrees with what is reported by Blondin \& Marks (1996) and Klein \& Woods (1998). They suggest a growth rate $\sim(k L)^{0.7}$. These respective growth rates of the NTSI, however, do not match with that proposed by Vishniac (1994) and given by Eq. (15) above. This equation predicts a much faster growth $\left(k^{1.5}\right)$, of the instability. In fact, the instability in this case has been observed to grow on a timescale comparable to the sound crossing time across the shocked slab.

We have argued that gas within the shocked slab is in turbulent motion, so that the slab is internally shocked and mechanical energy dissipated, leading to a loss of linear momentum of gas elements within the slab. The higher the viscosity, the greater the dissipation. We have already noted that growth of the NTSI is related to momentum transfer between perturbed regions of the slab. This dissipation should heat the gas up, a phenomenon that is obviated by a barytropic EOS. This is followed by lateral expansion of the gas slab, which soon becomes bloated. Rounding of the perturbed surfaces will reduce local pressure imbalances, another crucial factor inconducive to the growth of NTSI. Concurrence of these two factors may be responsible for arresting the growth of this instability. The NTSI is observed in models 2 and 3 and grows at a comparable rate in either case. That artificial viscosity is the only common factor between the two cases is therefore likely to have influenced its growth. Other cited literature here, however, does not discuss the possible reasons for the observed slow growth rate. Looking at the end product of this simulation, it may be suggested that such a collision is likely to produce a diffuse cloud that may collapse further and form a long filament. Gravitational clustering may commence in this filament.

\subsubsection{Off-centre cloud collision}

The shocked slab is internally stressed and thus exhibits a nonuniform velocity field. Internal shear renders the slab layers $\mathrm{KH}$ unstable. The picture in Fig. 9 is a column density plot of the slab soon after its formation. The slab forms a well-defined structure that is either filamentary or globular. Some small globules merge with other similar globules to form larger ones, while a few others are destroyed. As in model 3, the colliding clouds, hence the post-collision gas slab, are unconfined. The confining shocks can therefore propagate outward. Thus, the slab in this case as well, passes through the same phases as the one in the previous case. It also exhibits rotational motion about an axis orthogonal to the plane of collision ( $z$ axis). As the slab tumbles, two satellite lobes break off and move away radially. See the snapshot corresponding to $t=0.27$ Myr in Fig. 8 .

The slab dissipates thermal support through an internal shearing motion. Usami et al. (1995) provide a detailed analytic account of oblique shock-compressed slabs, according to which gravitational instabilities are suppressed in these slabs. Shearing motion increases the effective sound speed and therefore damps the growth rate of gravitational instability, which is probably exacerbated by the type of EOS employed in this work.

Further more, these authors also predict the end product of such slabs to be an elongated object, similar to the one we observed at the end of this calculation. We terminated this calculation after the formation of a filament in the plane of collision. The sequence of colliding clouds and then formation of the filament has been shown in a time sequence of column density plots in Fig. 8. We note that the slab evolves in this case on a much longer timescale than earlier models. This is presumably due to the additional support provided by the angular momentum associated with the slab rotation.

We notice some perturbations on the slab surface (see snapshots for $t=0.07 \mathrm{Myr}$ and $t=0.12 \mathrm{Myr}$ in Fig. 8), but there is no evidence of their amplification. The NTSI thus seems to be damped out. The Coriolis force acting on the rotating slab may suppress perturbations normal to the slab surface, in other words, the bending modes of the slab. This is, however, contrary to the findings of Whitworth et al. (1995) who simulated offcentre low-velocity cloud collision and found that the pressurecompressed oblique slab underwent gravitational fragmentation. However, with just a few thousand particles, it is unlikely that they would have been able to resolve any dynamical instability, including the gravitational instability.

\section{Conclusions}

Cloud collision plays an important role in the interstellar gas dynamics and is one of the mechanisms for dissipating energy. Sufficiently dense clumps resulting from fragmentation of the post-collision gas slabs become self-gravitating and spawn single/multiple star formation. A violent phenomenon like cloud collision also replenishes the pool of elements in the galactic disk. Post shock gas attains incredibly high temperatures $\left(\gtrsim 10^{4} \mathrm{~K}\right)$; however, if the shocked gas radiates efficiently, it cools rapidly and the temperature soon drops, sometimes even to the initial preshock temperature. Gas is further compressed by the shock, and postshock gas bodies may attain densities as high as $\sim 10^{4} \mathrm{~cm}^{-3}-10^{5} \mathrm{~cm}^{-3}$. Under favourable conditions, star formation may commence in these gas bodies. Thus, gas in the ISM goes through a cycle of phase transition starting from- diffuse gas to MCs to shocked slabs to star-forming cores to stars and back to diffuse gas. In some cases, however, there may be no star formation between the shock phase and the final diffused state.

The scope of the cloud collision problem is quite large, so here we attempt to investigate only its dynamical features. With the aid of the simple models tested in this work, we have attempted to suggest that cloud collision provides a viable mechanism for the formation of elongated structure in the ISM. 

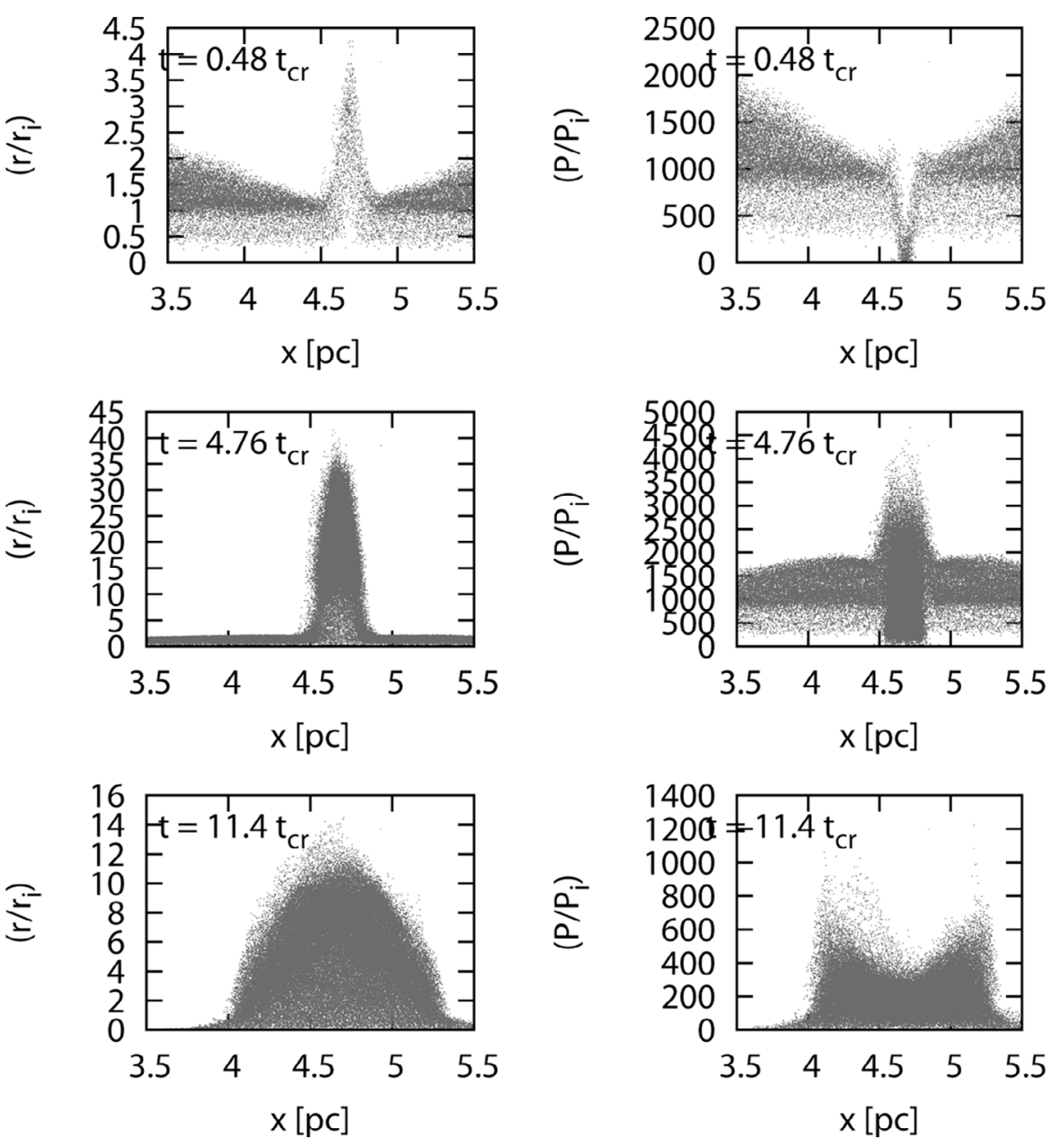

Fig. 11. Time evolution of density and pressure against distance along the collision axis in model 3. Time is measured in units of the cloud-crushing time, $t_{\mathrm{cr}}$. Both density and pressure have been normalised relative to their respective initial values. The top panel shows the post-collision jump in density and pressure. The shocked slab expands as the confining shocks move outward, leading to a drop in density and pressure within the slab. This can be seen in the second and third panels above.

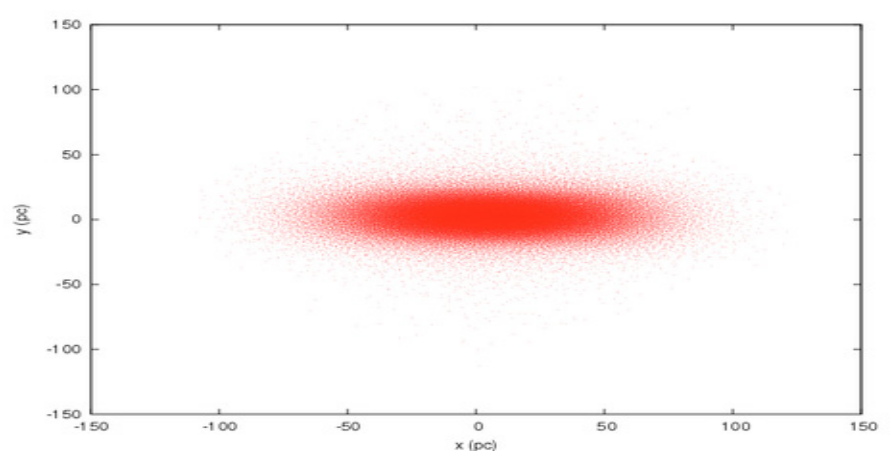

Fig. 12. A plot of particle positions showing the formation of an elongated object in model 3. At this epoch $(t=0.5 \mathrm{Myr})$, the shocked slab has expanded and undergone significant flattening.

The densest regions in these filaments may become self gravitating. We have concentrated only on the gravothermal aspect of the matter, thereby discounting the effect of the interstellar chemistry. We tried to ascertain the role of dynamical instabilities on the evolution of shocked gas slabs and have shown that there is much more to the problem than just a balance between gravity and thermal pressure. Figure 13 below is a plot of the normalised gravitational and thermal energy against time, for models 2, 3, and 4. A common feature of these plots is that the thermal energy rises steeply, post-shock, attains a maximum before rapidly falling off during the expansion phase of the slab. However, the shocked slab in model 2 only expands marginally and undergoes

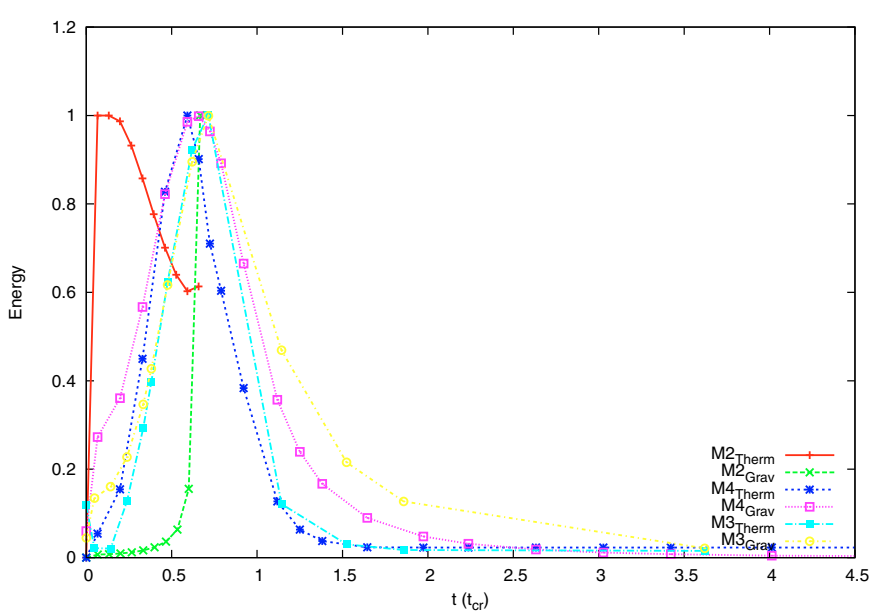

Fig. 13. A plot showing the time evolution of the normalised hydrodynamic and gravitational pressure in models 2,3 , and 4 . Time along the $x$ axis is marked in units of $t_{\text {cr }}$. This plot suggests that the shocked slab is supported against gravity unto the re-expansion phase, after which gravity starts dominating and the slab collapses. The key in the right hand corner lists the markers $\left(M_{\text {energy }}\right)$ for respective models.

gravitational fragmentation. The slab in this case is thermally supported, as is evident from the red curve in Fig. 13. The respective slabs in models 3 and 4 expand considerably and eventually end up at a temperature close to the one in the precollision clouds. The gravitational energy in models 3 and 4 is almost in 
sync with the thermal energy, with model 2 an exception. The cold shocked slab in this case becomes gravitationally unstable and fragments. The steep rise in gravitational energy corresponds to self-gravitating fragments.

In all the models tested here, the shocked slab becomes bloated soon after its formation, which is a unique feature of the NTSI. Two dynamical instabilities viz. the NTSI and the KH instability seem to play a vital role in energy dissipation via vortex formation within slab layers. Evidence for the NTSI has also been observed in grid simulations of colliding turbulent fluid flows (Heitsch et al. 2008). However, the gravitational instability dominates a shocked slab, provided it is sufficiently dense, or else it is suppressed and the slab expands. This can be seen in models 1, 3, and 4. Usami et al. (1995), for instance, make a similar claim. The NTSI appears to dominate the unconfined slab in model 3, while model 1 is poorly resolved, although the final result is the same. The slab in model 2, contained by the ICM, is more interesting as we observe the NTSI, competing with the gravitational instability and finally the slab fragments. The oblique shocked slab in model 4 develops kinks that seem to be damped, so nothing conclusive can be said about growth of the NTSI in this model.

Klein \& Woods (1998) and Heitsch et al. (2006) also report similar conclusions. The former additionally note that the NTSI is suppressed in adiabatic shocked slabs as such shocks do not radiate post-shock kinetic energy and therefore tend to rapidly expand on a much shorter timescale than required for the growth of bending modes. We measure the growth rate of the NTSI in one of our models, which agrees very well with that is reported in literature elsewhere.

Here we have adopted a simple EOS to account for radiative cooling and the assumption of an isothermal shock may have led to excessive post-shock compression. It is therefore essential to employ better cooling techniques (e.g. Koyama \& Inutsuka 2000; Vàzquez-Semadini et al. 2007). It is also essential to investigate the effect of SPH artificial viscosity on the growth rate of NTSI. We adopted the standard choice of viscous parameters in all our simulations. Our models are extremely simple, since we preferred to use physically identical clouds without any internal perturbations. Also, we did not included magnetic fields. It would be an instructive exercise to start with magnetised clouds and then collide them. We can then study the effect of magnetic field on the evolution of the shocked slab and, in particular, on the growth of various dynamical instabilities in a magnetised gas slab.

Acknowledgements. This work was completed as part of postgraduate research and funded by the State Government of Maharashtra, India (DSW/Edu/Inf/2004/6283(35)). I thank Dr. S. Goodwin for making the latest version of his SPH code DRAGON available. All the column density plots presented were prepared using the publicly available graphics package, SPLASH prepared by Dr. D. Price (Price 2007I). My gratitude to Prof A. Whitworth for suggesting this project. Useful comments and suggestions by an anonymous referee are greatly appreciated, and special thanks go to Prof. S. Falle for his critical observations on the original paper, which contributed immensely towards its improvement.

\section{References}

Agertz, Moore, B., Stadel, J., et al. 2007, MNRAS, 380, 963

Barnes, J., \& Hut, P. 1986, Nature, 324, 446
Bate, M., Bonnell, I., \& Price, N., 1995, MNRAS, 277, 362

Bhattal, Francis, N., Watkins, S. J., Whitworth, A. P., et al. 1998, MNRAS, 297, 435

Blitz, et al. 2007, Protostars and Planets V (Univ. Press Arizon), 951, 81

Blondin, J., \& Marks, B. 1996, New Astron., 1(3), 235

Burkert, A., \& Hartmann, L. 2004, ApJ, 616, 288

Chandrasekhar, S. 1939, An Introduction To The Study Of Stellar Structure (Dover Pub.)

Chandrasekhar, S. 1953, MNRAS, 113, 667

Chini, Reipurth, B., Ward-Thompson, D., et al. 1997, ApJ, 474, L135

Dale, J., Clark, P., \& Bonnell, I. 2007, MNRAS, 377, 535

Dehnen, W. 2001, MNRAS, 324, 273

Elmegreen, B. 1997, ApJ, 477, 196

Elmegreen, B. 2000, ApJ, 530, 277

Elmegreen, B. 2007, ApJ, 668, 1064

Fleck Jr., R. 1992, ApJ, 401, 146

Gingold, R., \& Monaghan, J. 1977, MNRAS, 181, 375

Goodwin, S., Whitworth, A., \& Ward-Thompson, D. 2004, MNRAS, 414, 633

Hartmann, L., Ballestros-Paredes, J., \& Bergin, E. 2001, ApJ, 562, 852

Hatchell, Richer, J. S., Fuller, G. A., et al. 2005, A\&A 440, 151

Hausman, M. 1981, ApJ, 245, 72

Heitsch, et al. 2006, ApJ, 642, 1052

Heitsch, M., Hartmann, L. W., Slyz, Adrianne D., Devriendt, J. E. G., Burkert, A., et al. 2008, ApJ, 674, 316

Hueckstaedt, R., Hartmann, L. W., Slyz, Adrianne D., Devriendt, J. E. G., Burkert, A., 2003, New Astron., 8, 295

Hunter, Sandford, M. T., II, Whitaker, R. W., Klein, R. I., et al. 1986, ApJ, 305, 309

Johnstone, D., \& Bally, J. 1999, ApJ, 510, L49

Klessen, R., \& Burkert, A. 2000, ApJS, 128, 287

Klessen, R., \& Burkert, A. 2001, ApJ, 549, 386

Klessen, R., Heitsch, F., \& McLow, M. 2000, ApJ, 526, 279

Klein, R., \& Woods, D. 1998, ApJ, 497, 777

Koyama, H., \& Inutsuka, S. 2000, ApJ, 532, 980

Kulkarni, S., \& Heiles, C. 1987, in Interstellar Processe (Dordecht Reidel Pub.), p. 87

Larson, R. 1981, MNRAS, 194, 809

Lattanzio, J. C., Monaghan, J. J., Pongracic, H., Schwarz, M. P., et al. 1985, MNRAS, 215, 125

Ledoux, P. 1951, An. Ap., 14, 438

Lucy, L. 1977, Aj, 82, 1013

McLow, M., \& Klessen, R. 2004, Rev. Mod. Phy., 76, 125

Mestel, L. 1965, QJRAS, 161

Miniati, F., Jones, T. W., Ferrara, A., Ryu, D., et al. 1997, ApJ, 491, 216

Nagai, T., Inutsuka, S., \& Miyama, S. 1998, ApJ, 506, 306

Padoan, P., \& Nordlund, Å. 1999, ApJ, 526, 279

Price, D. 2007, PASA, 24(3), 159

Price, D. 2007, [arXiv:0709.2772]

Raichaudhari, A. 1999, The Physics Of Fluids And Plasmas (Cambridge University Press)

Roberts, W. 1969, ApJ, 158, 123

Sasao, T. 1973, PASJ, 25, 1

Schneider, S., \& Elmegreen, B. 1979, ApJS, 41, 87

Shore, S. 2007, Astrophysical Hydrodynamics (John Wiley Pub. Inc.)

Simon, R. 1965, An. Ap, 28, 40

Solomon, et al. 1987, ApJ, 319, 730

Spitzer, L. 1968, Nebulae \& Interstellar Matter, ed. B. Middlehurst, \& A. Lawrence (Chicago University Press), Chap. 1

Spitzer, L. 1978, Physical Processes In The Interstellar Medium

Stevens, I., Blondin, J., \& Pollock, I. 1992, ApJ, 386, 265

Stone, M. 1970, ApJ, 159, 277

Stone, M. 1970, ApJ, 159, 293

Strickland, R., \& Blondin, J. 1995, ApJ, 449, 727

Usami, M., Hanawa, T., \& Fujimoto, M. 1995, PASJ, 47, 271

Vázquez-Semadeni, E., Gómez, G. C., Jappsen, A. K., et al. 2007, ApJ, 657, 870

Vietri, M., Ferrara, A., \& Miniati, F. 1997, ApJ, 483, 262

Vishniac, E. 1983, ApJ, 274, 152

Vishniac, E. 1994, ApJ, 428, 186

Whitworth, Chapman, S. J., Bhattal, A. S., et al. 1995, MNRAS, 277, 727 